\title{
Implantation of additional subcutaneous array electrode reduces defibrillation threshold in ICD patients - preliminary results
}

Maciej Kempa, Szymon Budrejko, Łukasz Drelich, Tomasz Królak, Grzegorz Raczak, Dariusz Kozłowski

Department of Cardiology and Electrotherapy, Medical University of Gdansk, Poland

Submitted: 12 January 2012

Accepted: 30 November 2012

Arch Med Sci 2013; 9, 3: 440-444

DOI: 10.5114/aoms.2013.35480

Copyright @ 2013 Termedia \& Banach

\begin{abstract}
Introduction: Among the recipients of implantable cardioverter-defibrillators (ICDs), there is a group of patients in whom the defibrillation threshold (DFT) is too high to enable a sufficient safety margin between the DFT and the maximal available output of the device. The aim of the study was to investigate the ability of an additionally implanted single-coil subcutaneous array electrode to reduce the DFT in such patients.

Material and methods: Medtronic 6996SQ electrode was implanted in 15 patients selected from our follow-up group of 741 ICD patients: 10 of them had insufficient post-implant DFT safety margin, and 5 had ineffective first maximal energy shock as recorded by the device. In 6 cases the patients had CRT-D devices, in 5 cases - dual-chamber ICDs, and in 4 cases - single-chamber ICDs. In all patients but one the defibrillating electrode was single-coil. In one patient it was dual-coil. The underlying disease was coronary artery disease in 10 patients, dilated cardiomyopathy in 4 patients and hypertrophic cardiomyopathy in 1 patient. Results: The subcutaneous electrode was successfully implanted in all the patients qualified for the procedure. No technical issues or perioperative complications were observed. Mean DFT was reduced from $33.3 \pm 4.1 \mathrm{~J}$ before the procedure to $25.3 \pm 4.4 \mathrm{~J}$ after the implantation procedure $(p<0.01)$.

Conclusions: Our results show that the use of a single-coil subcutaneous electrode to reduce the DFT is a safe and effective procedure. Further studies are necessary to confirm these results.
\end{abstract}

Key words: implantable cardioverter-defibrillator, implantable cardioverter-defibrillator, defibrillation threshold, subcutaneous electrode.

\section{Introduction}

Implantation of an implantable cardioverter-defibrillator (ICD) is a recognized method of prevention of sudden cardiac death in patients at risk for malignant ventricular arrhythmias. The essential condition is to deliver defibrillating shock energy high enough to exceed the defibrillation threshold (DFT) in a particular patient.

Modern defibrillating systems enable relatively low defibrillation thresholds to be achieved. It is due to the introduction of new lead models, as well as the use of an active ICD can and a biphasic defibrillating impulse [1-3]. But despite that, there is still a group of patients, in whom the maximal shock output of the implanted device fails to terminate ventricular fibrillation (VF), or the DFT is too high to enable a sufficient safety margin

\author{
Corresponding author: \\ Maciej Kempa MD, PhD \\ Department of Cardiology \\ and Electrotherapy \\ Medical University of Gdansk \\ 7 Debinki St \\ 80-211 Gdansk, Poland \\ Phone/fax: +48 583493910 \\ E-mail: kempa@gumed.edu.pl
}


between the DFT and the maximal available output of the device. The solution to such a situation may be the change of polarity of the defibrillating impulse or the use of intravascular electrodes with an additional defibrillating coil that is placed on the border of the superior caval vein and right atrium $[1,4,5]$. The use of a dual-coil defibrillating lead is possible only if a high DFT is diagnosed during the ICD implantation procedure. Changes in the DFT later in the postoperative period cannot be treated in such a manner, because it would require the extraction of the previously implanted single-coil lead, which becomes increasingly difficult the longer the period since the initial procedure.

In patients with a DFT increase late in the postoperative period or in patients in whom the above measures have failed, it is possible to implant an additional subcutaneous array electrode. Former studies proved that such a solution offers a mean DFT reduction of $20 \%$ to $60 \%$, depending on the electrode model [6-9].

\section{Material and methods}

In 2007-2010 an ICD (Biotronik, Medtronic) implantation procedure was carried out in $610 \mathrm{pa-}$ tients (78\% M, 22\% F, mean age $63 \pm 13$ years) and an ICD (Biotronik, Medtronic) reimplantation procedure in 131 patients (79\% M, 21\% F, mean age 62 \pm 12 years).

A defibrillation test was performed immediately after the surgical procedure or during the next 2-3 days. The test was performed in 675 patients. The test was not performed in 66 patients, when typical contraindications were present, such as: persistent atrial fibrillation or artificial valve without adequate anticoagulation, thrombi in heart chambers, hemodynamic instability, severe aortic stenosis, prior stroke, or symptomatic and severe coronary artery disease not eligible for revascularization [10]. Tests were performed in an electrophysiology laboratory dedicated to pacemaker and ICD implantation procedures.

After prior inspection of pacing and sensing parameters, ventricular fibrillation was induced once during short general anesthesia performed by an anesthesiologist. The shock-on-T or burst induction method was used. Each ICD was programmed to deliver the first shock of an energy lower by $10 \mathrm{~J}$ (max-10) or $15 \mathrm{~J}$ (max-15) than the maximal output of the particular ICD device model. The second shock was programmed with the maximal energy for the particular ICD model in the former case and the energy higher by $5 \mathrm{~J}$ in the latter case. Subsequent shocks were of maximal energy. The test was satisfactory if VF was terminated by the ICD with a shock of an energy of max-15 or max-10.

If the shock of the energy max-10 was not effective, that is if the $10 \mathrm{~J}$ safety margin could not be maintained, the arrhythmia was terminated with external defibrillation. In such a situation the test was repeated with reverse impulse polarity. If that method also failed to satisfy the $10 \mathrm{~J}$ safety margin criterion, the patient was qualified for a subcutaneous electrode implantation procedure.

\section{Statistical analysis}

Statistical analysis (Wilcoxon test) included the maximal energy of the ineffective ICD shock before the implantation of the subcutaneous electrode and the energy of the effective shock after the procedure. Inter-group difference with a test $p$ value of less than 0.05 was considered statistically significant.

\section{Subcutaneous electrode}

The subcutaneous array electrode Medtronic 6996SQ consists of a single defibrillating coil that is $25 \mathrm{~cm}$ long and has a diameter of $7.5 \mathrm{~F}$, and an electrical cord ending with a $3.2 \mathrm{~mm}$ connector type DF-1. Total length of the electrode is $41 \mathrm{~cm}$ or $58 \mathrm{~cm}$. That system is connected to the SVC socket of an ICD. If a dual-coil intravascular lead is used, the subcutaneous electrode may be connected through the Y-connector to the SVC socket together with the proximal coil of the intravascular lead.

\section{Implantation procedure of the 6996SQ electrode}

The implantation procedure of the 6996SQ electrode was performed in an operating room. Prior to the procedure informed consent was obtained from all patients. The patient was lying flat, with the abducted left upper limb and an additional support under the left scapula. Local anesthesia was applied in the ICD pocket and along the designed course of the subcutaneous electrode. Then the device was extracted. The tunneling tool 6996ST (provided by the manufacturer with the electrode) with a dedicated sheath on it was shaped appropriately and introduced into the ICD pocket and further into the subcutaneous tissue, from the lower margin of the pocket, along the chest wall, and towards the region below the inferior angle of the left scapula. Then the tunneling tool was removed and the electrode with an introducer inside was inserted into the sheath. Following that, the sheath was removed with an attached slittering tool, the introducer was removed from the inside of the electrode, and the electrode itself was sutured in the pocket in a manner typical for intravascular leads. The electrode was then connected to the SVC socket of the ICD. The pocket and the surgical wound were closed typically. Owing to that method, the elec- 


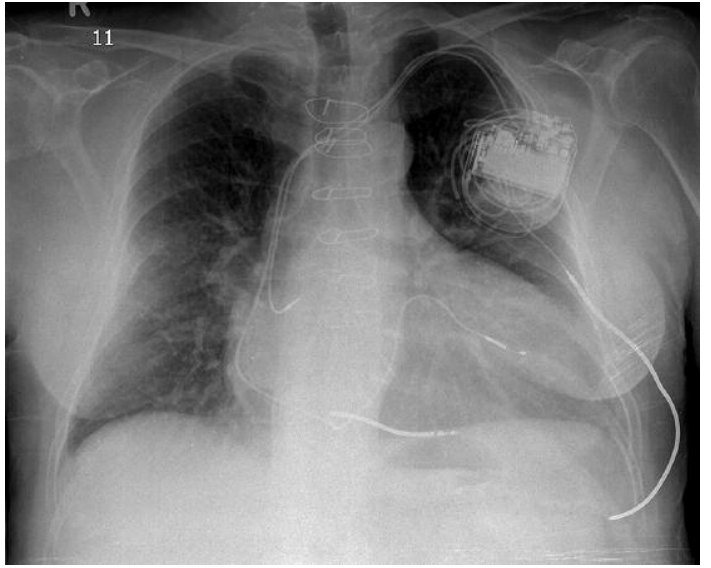

Figure 1. An X-ray image of a patient with a cardiac resynchronization therapy defibrillator system with a subcutaneous array electrode. Antero-posterior view

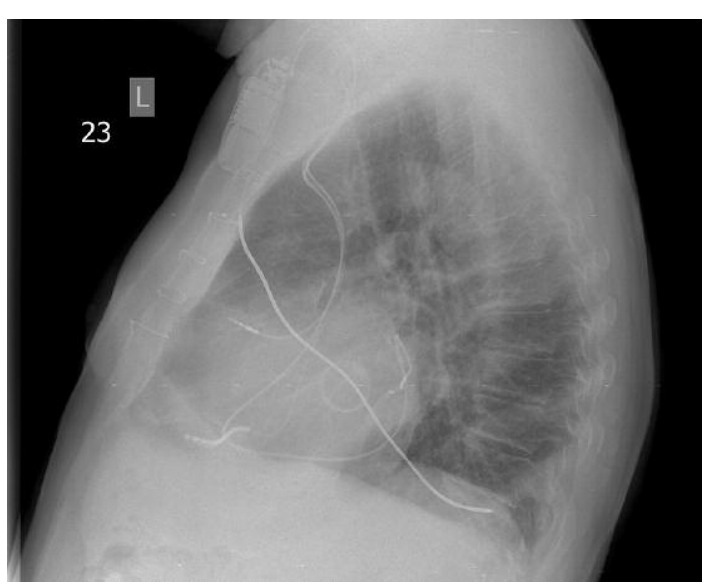

Figure 2. An X-ray image of a patient with a cardiac resynchronization therapy defibrillator system with a subcutaneous array electrode. Lateral view trode along its course remained in the projection of the chest, and its end was located as close to the vertebral column as possible. No additional incisions beyond the ICD pocket opening were performed (Figures 1 and 2). No complications of the subcutaneous electrode implantation procedure were observed in any of the cases.

\section{Results}

Among 675 patients subjected to an ICD test, in 19 cases $(2.8 \%)$ the shock of an energy max-10 failed to terminate the VF. In 9 patients the reversal of polarity allowed for the efficacy of the impulse max-10, and the remaining 10 patients were qualified for the implantation of a subcutaneous electrode. Moreover, during the study period the subcutaneous electrode was also implanted in 5 patients, in whom ineffective defibrillation with the first maximal energy impulse (for the particular ICD model) was observed during the late follow-up. In those cases the clinical arrhythmia was terminated with one of the subsequent shocks. Altogether, the subcutaneous electrode was implanted in $15 \mathrm{pa}$ tients ( $13 \mathrm{M}, 2 \mathrm{~F}$, mean age $56 \pm 10$ years). In 6 cases the patients had CRT-D devices, in 5 cases dualchamber ICDs, and in 4 cases single-chamber ICDs. In all patients but one, the defibrillating electrode was single-coil. In one patient it was dual-coil. The underlying disease was coronary artery disease in 10 patients, dilated cardiomyopathy in 4 patients and hypertrophic cardiomyopathy in 1 patient. The characteristics of the patients included in the study are summarized in Table I.

Statistical analysis (Wilcoxon test) included the maximal energy of the ineffective ICD shock before the implantation of the subcutaneous electrode (10 patients) (DFT-SQ) and the energy of the effective shock after the procedure (DFT+SQ). In patients with late shock inefficacy, the analysis included the data of the ineffective shock that was the basis for qualification for the subcutaneous electrode implantation procedure, and those data were extracted from the ICD memory (5 patients).

Mean DFT-SQ was $33.3 \pm 4.1 \mathrm{~J}$ and mean DFT+SQ was $25.3 \pm 4.4 \mathrm{~J}(p=0.0022)$ (Figures 3 and 4$)$. In one patient the ICD test after the subcutaneous electrode implantation procedure was not performed due to contraindications. Specific values of the defibrillation thresholds for each patient are listed in Table II.

\section{Discussion}

Both the correct detection and termination of arrhythmias are essential for any ICD in order to achieve its efficacy in terms of prevention of sudden cardiac death. A major purpose of ICD testing is to ensure the correct function of the device in that aspect. An ICD test allows one to assess sensing of the VF signal by the ICD, as well as to confirm the efficacy of a shock. Many authors

Table I. Characteristics of the patients with a subcutaneous electrode included in the study

\begin{tabular}{|lcrccc|}
\hline Number of patients & Sex & Age & Type of device & Type of defibrillating electrode & Underlying heart disease \\
\hline 15 & 13 male, & $56 \pm 10$ & $6-$ CRT-D; & 14 - single-coil; & 10 patients -CAD; \\
& 2 female & years & $5-$ ICD DR; & 1- dual-coil & 4 patients - DCM; \\
& & & $4-$ ICD VR & & 1 patient - HCM \\
\end{tabular}

$C A D$ - coronary artery disease, CRT-D - cardiac resynchronization therapy cardioverter-defibrillator, DCM - dilative cardiomyopathy, HCM - hypertrophic cardiomyopathy, ICD-DR-dual-chamber cardioverter-defibrillator, ICD-VR single-chamber cardioverter-defibrillator 


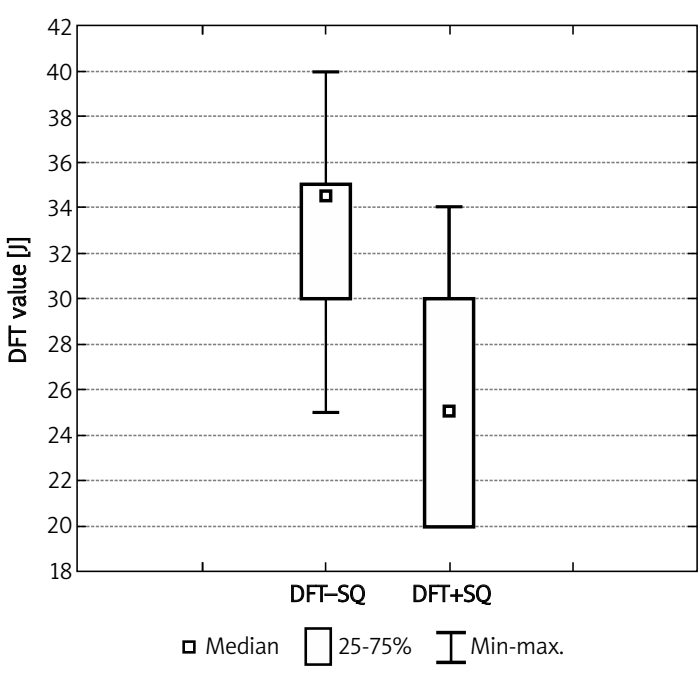

Figure 3. Comparison of the defibrillation threshold before and after implantation of a subcutaneous array electrode

DFT - defibrillation threshold, DFT-SQ - defibrillation threshold before the procedure, DFT+SQ - defibrillation threshold after the procedure

question the validity of ICD testing; nonetheless there is still a group of supporters of such a procedure [11-13].

In our cohort of patients the defibrillation test was performed routinely, unless contraindications were present. If the test failed to confirm the $10 \mathrm{~J}$ shock safety margin or if during late follow-up an inefficient shock of maximal energy was observed, the patient was referred for subcutaneous electrode implantation, associated with reimplantation of an ICD with higher maximal energy output if necessary. Such a procedure allowed the desired safety margin of at least $10 \mathrm{~J}$ or - in one case $-15 \%$ of the maximal available shock energy output (6 J) to be attained. The efficacy of that procedure in our observation is concordant with other reports. Analogically, the incidence of high DFT is similar, although lower, than the incidence reported in other studies $[13,14]$. In our study a high DFT was defined as higher than the maximal shock energy minus $10 \mathrm{~J}$. As different device models were used, in some patients a high DFT was defined as higher than $25 \mathrm{~J}$, and in others, higher than $30 \mathrm{~J}$. In the literature a high DFT was defined as more than $18 \mathrm{~J}$, especially in the period when the maximal available shock energy did not exceed $30 \mathrm{~J}$. Thus the incidence of such a condition may vary among different publications.

It is worth underlining that in our cohort of patients in as many as 5 cases we observed the late maximal shock inefficacy. It occurred despite correct results of the ICD test, that had been performed directly after the initial implantation procedure in 4 patients. In one case the ICD test had not been performed. That fact may confirm low

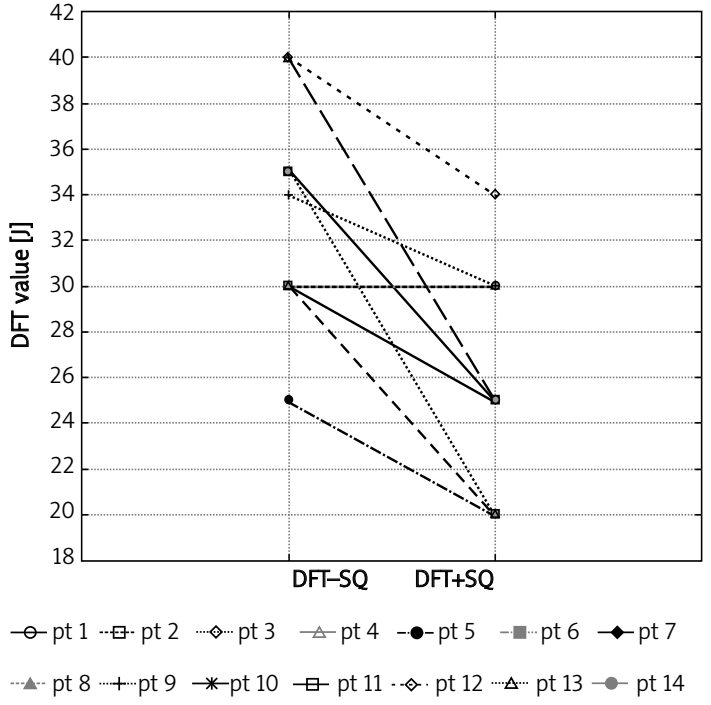

Figure 4. Values of the defibrillation threshold in successive patients in the cohort.

DFT - defibrillation threshold, DFT-SO - defibrillation thresh old before the procedure, DFT+SQ - defibrillation threshold after the procedure

reproducibility of ICD testing results, as suggested by some authors, which questions the rationale of its use. Guenther et al. underline the fact that due to the limited reproducibility of the ICD test results, in specific subgroups of patients the test should be performed twice: intraoperatively and prior to discharge from the hospital. In their population of 783 patients, in whom the efficacy of defibrillation

Table II. Specific values of the defibrillation thresholds for each patient

\begin{tabular}{|c|c|c|}
\hline Patient & DFT-SQ & $\mathrm{DFT}+\mathrm{SQ}$ \\
\hline 1 & $>30$ & 30 \\
\hline 2 & $>35$ & 25 \\
\hline 3 & $>35$ & 20 \\
\hline 4 & $>40$ & 25 \\
\hline 5 & $>25$ & 20 \\
\hline 6 & $>35$ & No test \\
\hline 7 & $>30$ & 20 \\
\hline 8 & $>30$ & 25 \\
\hline 9 & $>30$ & 30 \\
\hline 10 & $>34$ & 30 \\
\hline 11 & $>35$ & 25 \\
\hline 12 & $>35$ & 25 \\
\hline 13 & $>40$ & 34 \\
\hline 14 & $>30$ & 20 \\
\hline 15 & $>35$ & 25 \\
\hline
\end{tabular}

DFT-SQ - defibrillation threshold prior to implantation of the subcutaneous electrode, DFT+SQ - defibrillation threshold with the subcutaneous electrode implanted 
was confirmed intraoperatively with a safety margin of $>10 \mathrm{~J}$, in 9 cases the repeated test failed to confirm such a safety margin, and such patients required additional interventions (e.g. implantation of a subcutaneous electrode) [15].

Because of the retrospective nature of our study and lack of some data, we did not analyze the risk factors for high DFT occurrence in that cohort. That issue was analyzed and discussed in one of our other studies in a different population, as well as in numerous studies of other groups $[14,16]$.

Our study confirmed the safety of subcutaneous electrode use. The authors of other studies also stress that the risk of single-coil subcutaneous electrode implantation is low. The most frequent reported complication is pneumothorax $[13,14]$. In our cohort no complications were observed.

The limitation of that study is the relatively low number of patients in this cohort. But one should consider the fact that in Poland the first single coil subcutaneous electrode was implanted as recently as in 2004. Earlier single-case experiences were based on the use of triple-coil electrodes. Our cohort of 15 patients undergoing the procedure of singlecoil subcutaneous electrode implantation, performed in a single center, by the same operating team, and according to the same routine, is by far the most numerous described in Poland.

In conclusion, our results prove that the use of a single-coil subcutaneous defibrillating electrode to reduce the DFT of VF is a safe and effective procedure. Further studies are necessary to confirm these results.

\section{References}

1. Gold MR, Olsovsky MR, Pelini MA, Peters RW, Shorofsky SR. Comparison of single- and dual-coil active pectoral defibrillation. J Am Coll Cardiol 1998; 31: 1391-4.

2. Kavanagh KM, Tang AS, Rollins DL, Smith WM, Ideker RE. Comparison of the internal defibrillation threshold for monophasic and double and single capacitor biphasic waveforms. J Am Coll Cardiol 1989; 14: 1343-9.

3. Saksena S, Scott SE, Accorti PR, Boveja BK, Abel D, Callaghan FJ. Efficacy and safety of monophasic and biphasic waveform shock using braided endocardial defibrillation lead system. Am Heart J 1990; 120: 1342-7.

4. Shorofsky SR, Gold MR. Effects of waveform and polarity on defibrillation threshold in humans using a transvenous lead system. Am J Cardiol 1996; 78: 313-6.

5. Schauerte P, Stellbrink C, Schoendube FA, et al. Polarity reversal improves defibrillation efficacy in patients undergoing transvenous cardioverter defibrillator implantation with biphasic shock. Pacing Clin Electrophysiol 1997; 20: 301-6.

6. Kuhlkamp V, Khalighi K, Dornberger V, Ziemer G. Singleincision and single-element array electrode to lower the defibrillation threshold. Ann Thorac Surg 1997; 64: 1177-9.

7. Higgins SL, Alexander DC, Kuypers CJ, Brewster SA. The subcutaneous array: a new lead adjunct for the transvenous ICD to lower defibrillation threshold. Pacing Clin Electrophysiol 1995; 18: 1540-8.
8. Kuhlkamp V, Dornberger V, Khalighi K, Mewis C, Suchalla R, Ziemer G. Effect of a single element subcutaneous array electrode added to a transvenous electrode configuration on the defibrillation field and the defibrillation threshold. Pacing Clin Electrophysiol 1998; 21: 2596-605.

9. Gradaus R, Block M, Seidl K, et al. Defibrillation efficacy comparing a subcutaneous array electrode versus an active can implantable cardioverter defibrillator and a subcutaneous array electrode in addition to an active can implantable cardioverter defibrillator. J Cardiovasc Electrophysiol 2001; 12: 921-7.

10. Pires LA. Defibrillation testing of the implantable cardioverter defibrillator: when, how, and by whom? Indian Pacing Electrophysiol J 2007; 7: 166-75.

11. Michowitz Y, Lellouche N, Contractor T, et al. Defibrillation threshold testing fails to show clinical benefit during longterm follow-up of patients undergoing cardiac resynchronization therapy defibrillator implantation. Europace 2011; 13: 683-8.

12. Sauer WH, Lowery CM, Bargas RL, et al. Utility of postoperative testing of implantable cardioverter-defibrillators. Pacing Clin Electrophysiol 2011; 34: 186-92.

13. Osswald BR, De Simone R, Most S, Tochtermann U, Tanzeem A, Karck M. High defibrillation threshold in patients with implantable defibrillator: how effective is the subcutaneous finger lead? Eur J Cardiothorac Surg 2009; 35: 489-92.

14. Verma A, Kaplan AJ, Sarak B, et al. Incidence of very high defibrillation thresholds (DFT) and efficacy of subcutaneous (SQ) array insertion during implantable cardioverter defibrillator (ICD) implantation. J Interv Card Electrophysiol 2010; 29: 127-33.

15. Guenther M, Rauwolf T, Bruggemann B, et al. Pre-hospital discharge testing after implantable cardioverter defibrillator implantation: a measure of safety or out of date? A retrospective analysis of 975 patients. Europace 2012; 14: 217-23.

16. Lubiński A, Lewicka-Nowak E, Zienciuk A, et al. Clinical predictors of defibrillation threshold in patients with implantable cardioverter-defibrillators. Kardiol Pol 2005; 62: 317-28. 